\title{
Le Même et l'Autre : la question du métissage dans l'œuvre « conjecturale » de J. H Rosny aîné
}

\section{François Laforge}

\section{(2) OpenEdition}

\section{Journals}

Electronic version

URL: http://journals.openedition.org/belphegor/899

DOI: $10.4000 /$ belphegor.899

ISSN: 1499-7185

Publisher

LPCM

Electronic reference

François Laforge, « Le Même et l'Autre : la question du métissage dans l'œuvre « conjecturale » de J. H Rosny aîné », Belphégor [Online], 15-1 | 2017, Online since 20 June 2017, connection on 19 April 2019. URL : http://journals.openedition.org/belphegor/899; DOI : 10.4000/belphegor.899

This text was automatically generated on 19 April 2019

\section{cc)}

Belphégor est mis à disposition selon les termes de la Licence Creative Commons Attribution - Pas d'Utilisation Commerciale - Pas de Modification 4.0 International. 


\title{
Le Même et l'Autre : la question du métissage dans l'œuvre « conjecturale » de J. H Rosny aîné
}

\author{
François Laforge
}

1 Un certain nombre d'études ont souligné le rôle central du métissage dans la pensée et l'imaginaire de Rosny, comme dans son écriture. E. Lysoe parle ainsi, à son propos, d'une "poétique du métissage ", où il décèle « le goût de Rosny pour le mélange [...] qui fait de lui l'un des plus ardents défenseurs du métissage, l'un des plus singuliers poètes de l'impur $»^{1}$. Il montre comment l'écriture de Rosny associe une pratique systématique de l'hybridation générique, brouillant les frontières entre formes littéraires, à une thématique récurrente de la rencontre entre univers étrangers, dont le modèle paradigmatique est celui de la conjonction sexuelle sous ses différentes modalités, directes ou euphémisées ${ }^{2}$. La notion de métissage, dans le sens très large que lui donne $\mathrm{E}$. Lysoe, apparaît ainsi comme une clé de compréhension fondamentale d'une œuvre par ailleurs énigmatique dans sa diversité et son apparente dispersion.

2 Nous nous intéresserons ici au métissage au sens restreint du terme, comme processus biologique de croisement entre « races » ou espèces différentes ${ }^{3}$, voire entre formes de vie étrangères les unes aux autres, Rosny donnant à ce processus, comme nous le verrons, une extension très supérieure à celle qui est généralement admise par la science. Nous voudrions nous interroger sur la signification de ce thème, récurrent dans ses récits, notamment par rapport à la question, sensible pour le lecteur moderne, du racisme, réel ou supposé, de leur auteur. On le lui a souvent reproché $e^{4}$ même si l'on y reconnaît un trait d'époque, imputable à l'influence d'une idéologie alors largement répandue, qui trouvait dans la science contemporaine une caution apparemment définitive. Comment interpréter, dès lors, la présence constante dans les textes narratifs de Rosny de références au métissage et à la "fusion des races", pour reprendre une expression souvent utilisée par lui ${ }^{5}$ ? Faut-il y voir un désaveu, au moins partiel, de toute idéologie raciale ou « racialiste $»^{6}$, repliée sur l'affirmation des identités biologiques et la nécessité de les préserver, et un plaidoyer en faveur d'une pensée ouverte sur l'Altérité ? Nous 
essaierons de montrer, contre une telle interprétation, qui a été soutenue par un certain nombre de commentateurs ${ }^{7}$, soucieux d'exempter Rosny d'un racisme même "naïf ", difficilement acceptable pour le lecteur moderne, que sa position reste marquée par une ambiguïté fondamentale, perceptible dans un certain décalage, au niveau du traitement narratif du thème, entre le discours explicite des textes et leur fonctionnement effectif, entre ce qu'ils disent et ce qu'ils montrent, pour reprendre une distinction éclairante de M. Guillaumie ${ }^{8}$. Cette ambiguïté apparaît comme le reflet des tensions internes d'une pensée et d'un imaginaire partagés entre des postulations idéologiques contradictoires, qui renvoient elles-mêmes à un certain nombre d'oppositions fondamentales structurant la vision du monde de Rosny.

3 Nous nous attacherons plus particulièrement dans ce travail à l'analyse du thème du métissage dans les textes de Rosny relevant de la littérature "conjecturale $»^{9}$ (fictions "préhistoriques", récits de "mondes perdus», "merveilleux scientifique»), à l'exclusion des récits réalistes et des essais philosophiques ou de vulgarisation scientifique. Le choix de ce corpus restreint se justifie par la plus grande lisibilité des thèmes idéologiques dans ces récits libérés des contraintes réalistes, qui jouent au contraire pleinement dans les études "de mœurs ", où la thématique "raciale " prend une forme moins explicite, même si elle reste présente à l'arrière-plan des analyses psychologiques ou sociales de l'auteur. Cette lisibilité accrue tient notamment au fait que la question, sous-jacente au thème du métissage, des rapports du Même et de l'Autre se trouve posée de façon particulièrement aiguë dans ces textes, où l'altérité de l'Autre prend volontiers la forme radicale de l'étrangeté, voire de la monstruosité. Les œuvres de littérature « conjecturale » fonctionnent, en ce sens, comme des révélateurs privilégiés de l'idéologie de l'auteur, mais aussi des contradictions de celle-ci. De là, leur intérêt majeur pour notre propos ${ }^{10}$.

4 Les textes « conjecturaux » de Rosny mettent en scène de nombreux couples «mixtes », appartenant à des populations plus ou moins hétérogènes, comme le montre le tableau suivant. Pour plus de clarté, nous avons classé les œuvres concernées en plusieurs grandes catégories: récits "préhistoriques", récits de "mondes perdus", récits de «merveilleux scientifique », sans méconnaître le caractère arbitraire d'un tel classement, encore accentué par la pratique de l'hybridation générique, caractéristique de l'écriture de Rosny, comme nous l'avons vu. Nous avons, par ailleurs, inclus dans notre relevé aussi bien les couples mixtes effectifs, unis par une relation physique réelle, que ceux qui ont une simple existence virtuelle, comme le couple formé, dans Helgvor du fleuve bleu, par le chef Gwah et Glâva, ou celui que constituent l'Homme-des-eaux et Sabine dans Nymphée.

\begin{tabular}{|c|c|c|c|}
\hline \multicolumn{4}{|c|}{ Récits «préhistoriques» } \\
\hline Vamireh & roman & 1892 & Pzann + femme Asiatique \\
\hline Elem d'Asie ${ }^{11}$ & nouvelle & 1896 & Pzann + femme Asiatique \\
\hline Eyrimah & roman & 1896 & $\begin{array}{l}\text { Montagnard + femme Lacustre } \\
\text { Lacustre + Montagnarde }\end{array}$ \\
\hline Le Félin géant & roman & 1920 & Oulhamr + «Femme-louve » \\
\hline
\end{tabular}




\begin{tabular}{|c|c|c|c|}
\hline Helgvor du fleuve bleu & roman & 1930 & $\begin{array}{l}\text { Ougmar + femme Tzoh } \\
\text { Gwah + femme Tzoh }\end{array}$ \\
\hline \multicolumn{4}{|l|}{ Récits de $«$ mondes perdus $»^{12}$} \\
\hline Nymphée & roman & 1893 & « Homme-des-eaux » + Européenne \\
\hline Les Femmes de Setnê & roman & 1903 & Egyptien + «Femme-des-eaux » \\
\hline L'Etonnant voyage de Hareton Ironcastle & roman & 1922 & « Homme-Trapu »+ Européenne \\
\hline Le Trésor dans la neige & nouvelle & 1922 & Européen + « Touranienne » \\
\hline La Fille bleue & nouvelle & 1927 & Européens + «filles bleues » \\
\hline Les Hommes sangliers & nouvelle & 1929 & « Homme-sanglier »+ Européenne \\
\hline La Résurrection de mon oncle Jérôme & nouvelle & 1933 & Européen + « Magdalénienne » \\
\hline La Sauvage aventure ${ }^{13}$ & roman & 1935 & « Homme-Kérabau » + Européenne \\
\hline \multicolumn{4}{|l|}{ Récits de « merveilleux scientifique » } \\
\hline Un autre monde & nouvelle & 1898 & Mutant ( ?) + humaine \\
\hline La Jeune vampire & nouvelle & 1920 & Humain + femme « vampire » \\
\hline Les Navigateurs de l'infini & roman & $1925 / 1960$ & Terrien + Tripède martienne \\
\hline Dans le monde des variants & nouvelle & 1939 & Humain-Variant + Variante \\
\hline
\end{tabular}

5 La lecture de ce tableau montre que tous les genres de littérature « conjecturale » sont concernés par le thème du métissage, ce caractère transversal soulignant sa prégnance dans l'imaginaire de Rosny, également mise en évidence par sa remarquable persistance tout au long de la carrière de l'auteur. Un premier ensemble de textes est constitué par les récits " préhistoriques ", où le métissage apparaît comme un motif narratif récurrent, présent dans la quasi-totalité du cycle, qui trouve là un élément important d'unité et de cohérence, comme l'ont souligné plusieurs commentateurs ${ }^{14}$. Deux textes font cependant ici exception : Nomaï, une courte nouvelle publiée en 1897, qui raconte la naissance de l'Amour à travers l'invention du baiser, et, surtout, La Guerre du feu (1911), qui occupe une place centrale dans l'ensemble, tant par sa situation chronologique que par sa richesse thématique et narrative. Contrairement aux autres récits du cycle, où le héros s'unit à une femme étrangère à son peuple ou à sa " race ", ces deux œuvres privilégient l'une et l'autre des relations de type endogamique: Nomaï et Amreh, les deux protagonistes de Nomaï, appartiennent en effet à la même tribu; il en va de même pour Naoh, le personnage principal de La Guerre $d u$ feu, et Gammla, la femme qu'il obtient au terme de sa quête. Une sorte de fermeture identitaire semble ainsi se substituer à l'ouverture fusionnelle à l'Autre, privilégiée dans les autres œuvres du cycle. On doit cependant souligner que cette fermeture est compensée, dans les deux récits, par la valorisation d'autres formes de relation à l'Autre, qu'il s'agisse de sentiments altruistes, comme 
l'amour ou la pitié, ou de pratiques de métissage culturel, comme dans La Guerre du feu, où le personnage principal emprunte à la tribu des Hommes-sans-épaules un certain nombre de techniques qu'il fera adopter par son peuple ${ }^{15}$.

Un second ensemble de textes, le plus important quantitativement, est constitué par les récits de "mondes perdus", qui accordent également une place centrale au thème du métissage, ce qui confirme, s'il en était besoin, la proximité de ce type de récits avec les romans "préhistoriques ». Le motif apparaît, là encore, dans la plupart des œuvres relevant de ce genre, à quelques exceptions près, comme Les Profondeurs de Kyamo (1896) ou La Grande énigme (1920). L'absence du thème du métissage dans ces textes a cependant une signification différente de celle qu'elle pouvait avoir dans les récits "préhistoriques ", analysés précédemment. Elle apparaît, en effet, essentiellement liée à la disparition de toute thématique érotique ou affective. Tout se passe, d'une certaine façon, comme si la dimension érotique ou sexuelle était nécessairement associée, dans ce type de récit, à la mise en scène d'une sexualité exogamique et au métissage. Une autre caractéristique des récits de "mondes perdus » est de décliner le thème du métissage selon deux versions : tantôt c'est un homme moderne, généralement un Européen, qui s'unit avec une ou plusieurs femmes appartenant à l'un de ces mondes, comme dans Le Trésor dans la neige, tantôt c'est une Européenne qui s'unit, le plus souvent sous la contrainte (viol, enlèvement, etc.), à un homme "primitif», comme dans Les Hommes sangliers ou La Sauvage aventure. La tonalité générale du récit varie sensiblement d'une version à l'autre, plus euphorique dans un $\operatorname{cas}^{16}$, plus dramatique dans l'autre. Il suffira de rappeler ici le dénouement tragique des Hommes sangliers, qui se concluent par le suicide de l'héroïne, traumatisée par le viol qu'elle a subi et les sentiments troubles qu'elle éprouve à l'égard de son persécuteur.

7 Le dernier ensemble de textes est constitué par les récits relevant du «merveilleux scientifique ». Le thème du métissage y apparaît moins fréquent que dans les deux autres types de récits, probablement parce que la plupart des textes de Rosny appartenant à ce genre mettent en scène des êtres totalement étrangers à l'humanité, voire à toute forme de vie animale, comme les Xipéhuz ou les Ferromagnétaux, quand ils n'appartiennent pas à un autre univers que le nôtre, tels les êtres lumineux décrits dans La Force mystérieuse. La distance avec l'homme est alors telle que l'idée même de métissage biologique devient impensable. On peut cependant souligner le fait que, même dans ces cas extrêmes, Rosny maintient la possibilité d'une sorte de métissage symbolique, qui apparaît comme le substitut d'une impossible fusion entre des formes de vie séparées les unes des autres par des barrières infranchissables. C'est clairement le sens du geste de Targ, à la fin de La Mort de la terre, qui choisit de mourir parmi les Ferromagnétaux pour transmettre à « la Vie Nouvelle " «quelques parcelles de la dernière vie humaine» (RSF, p. 188). On reste néanmoins ici sur le plan du symbole, ce qui donne à la décision du personnage son caractère à la fois pathétique et dérisoire. Seuls des êtres ayant avec l'homme une certaine analogie, même lointaine, pourront entrer dans un véritable processus de métissage biologique. Rosny repousse cependant très loin les limites de l'analogie. Si les Tripèdes martiens peuvent en effet offrir une vague ressemblance avec l'être humain, il n'en va pas de même pour les Variants, qui donnent son titre à la nouvelle Dans le monde des variants. Ils constituent une forme de vie réellement singulière, dont les particularités sont longuement décrites dans le récit ${ }^{17}$. La ressemblance avec l'homme se limite alors à quelques traits généraux, communs aux deux « espèces ", et qui constituent, en quelque sorte, les conditions minimales nécessaires à l'établissement d'une relation de métissage : 
existence d'une vie individuelle consciente, différenciation sexuelle et enfin présence de sentiments psychologiques complexes comme le désir ou l'amour.

8 Le spectre couvert par la notion de métissage apparaît ainsi extrêmement large chez Rosny. Il va du mélange de "races » faisant partie de la même espèce, comme dans les récits "préhistoriques", au métissage d'êtres appartenant à des espèces totalement différentes, voire à des formes de vie et à des univers différents, comme dans les récits de "merveilleux scientifique ", en passant par le croisement d'individus issus d'espèces distinctes, mais proches les unes des autres. Ce dernier cas de figure apparait dans un certain nombre de récits de "mondes perdus", qui mettent en scène des peuples primitifs étranges, présentés comme autant d'humanités autres: Hommes-sangliers, Hommes-Kérabaus, Hommes-des-eaux, Hommes-Trapus, etc. Le statut de ces populations reste quelque peu problématique chez Rosny, qui parle indifféremment à leur propos de «races » ou d' "espèces $»^{18}$. Elles n'en représentent pas moins d'autres possibilités de développement de l'espèce humaine, qui sont expliquées tantôt par les aléas de l'évolution biologique, comme dans Nymphée, où le peuple des eaux rencontré par le narrateur et ses compagnons est présenté comme le résultat d'une adaptation progressive à des conditions d'existence particulières ${ }^{19}$; tantôt par une origine différente, comme dans $\mathrm{La}$ Sauvage aventure, où Rosny, reprenant certaines théories polygénistes, déjà fortement contestées à son époque, suggère que les Hommes-kérabaus sont issus d'autres ancêtres anthropoïdes que ceux qui ont donné naissance à notre lignée ${ }^{20}$. Ainsi conçu, le métissage dépasse très nettement les limites que lui assignait la science de l'époque, comme celles que lui assigne la science moderne. Ce qui souligne la dimension fantasmatique de ce thème chez Rosny, dont les références scientifiques ou pseudoscientifiques dissimulent mal le rêve de fusion plus ou moins totale avec un Autre, aussi fascinant qu'inquiétant dans son étrangeté.

Quelle que soit la forme prise par le métissage dans ses récits, il obéit toujours au même schéma fondamental, qui associe un personnage symbolisant le Même, généralement le personnage principal ou le narrateur, et un second personnage placé sous le signe de l'Altérité. Ce que l'on pourrait résumer par la formule: MEME + AUTRE, chaque sexe pouvant indifféremment assumer l'un des deux rôles, même si l'Altérité est plus souvent associée à la féminité, comme cela a été souvent notée ${ }^{11}$. Cette formule souligne le fait que, dans les couples mixtes imaginés par Rosny, l'un des personnages appartient à un monde que le lecteur perçoit comme plus ou moins proche du sien, contrairement à l'autre, qui apparaît comme étranger à ce monde et, par là même, différent. Ce qui entraîne une identification immédiate avec le premier, encore favorisée par le jeu des focalisations et des voix narratives. Le point de vue adopté dans le récit est en effet généralement celui du personnage censé représenter le Même, qui se voit également chargé de la narration, lorsqu'il s'agit d'un récit homodiégétique ${ }^{22}$. Un Autre monde constitue, de ce point de vue, une exception remarquable, dans la mesure où c'est l'Autre qui assure ici la fonction de narrateur. Ce choix narratif audacieux introduit une distorsion entre l'altérité objective du personnage, une sorte de "mutant ${ }^{23}$ aux facultés étonnantes, et le sentiment de proximité suscité chez le lecteur par le mode de narration autodiégétique, qui, en nous donnant accès à son monde intérieur, crée les conditions d'une relation d'empathie. Le lecteur est ainsi amené à s'identifier à un être qu'il ressent pourtant comme fondamentalement autre, ce qui ne laisse pas d'être problématique. Il en résulte un certain brouillage du Même et de l'Autre, encore accentué par le fait que le personnage symbolisant le Même est une folle, "une pauvre fille hystérique» (RSF, p. 46), dont 
l'univers mental nous reste, par ailleurs, inaccessible, en l'absence de toute focalisation sur elle. On aboutit ainsi à ce résultat paradoxal et profondément troublant que le Même nous est, en un sens, plus étranger que l'Autre. Ce cas de figure reste cependant exceptionnel chez Rosny qui s'en tient, le plus souvent, à un mode de narration permettant d'identifier sans ambiguïté chacun des personnages.

La perception du métissage se trouve ainsi organisée selon un système d'oppositions implicite, lui-même porteur de jugements de valeurs, entre le proche et le lointain, le familier et l'étrange, le rassurant et l'inquiétant. Cette structure dissymétrique place le Même au centre de son dispositif, lui attribuant par-là même un rôle privilégié de référence obligée. Il est significatif, de ce point de vue, que Rosny s'intéresse peu au métissage entre "races" ou espèces exotiques. S'il lui arrive d'en parler, c'est généralement sur un mode allusif. Un court passage dans Nymphée signale ainsi au lecteur que « l'on découvre des traces de fusion » entre les différentes races d'Hommes-des-Eaux qui vivent au sein du monde souterrain découvert par le narrateur et ses compagnons ( RSF, p. 231). Nous n'en saurons pas davantage. Cette information ne joue, de toute façon, aucun rôle dans le récit, qui accorde au contraire une place centrale à la question d'un métissage possible entre les Hommes-des-Eaux et les Européens, à travers l'épisode dramatique de l'enlèvement de Sabine Devreuse. Cette différence de traitement montre que le thème du métissage n'a d'intérêt, pour Rosny, qu'à partir du moment où il fait intervenir le Même, avec les possibilités d'identification qu'il autorise. Sans la présence de cet élément, le thème n'éveille que peu d'échos dans l'imaginaire de Rosny. Ici, comme ailleurs, c'est moins l'Autre en lui-même que la rencontre avec l'Autre et les bouleversements affectifs et intellectuels qu'elle entraîne qui retiennent son attention, le métissage s'inscrivant par là dans la perspective générale du modèle construit par $\mathrm{E}$. Lysoe $^{24}$. La formule : MEME + AUTRE se trouve ainsi clairement privilégiée par Rosny par rapport à d'autres formules possibles.

11 Rosny choisit, pour symboliser le Même, un personnage aussi proche que possible de son lecteur ou, du moins, de l'image qu'il s'en fait. Il s'agit généralement d'un Européen ou d'une Européenne, vivant à l'époque moderne, soit la fin du XIXe siècle ou le début du siècle suivant. C'est le cas dans la plupart des récits de «merveilleux scientifiques » et de «mondes perdus », à l'exception des Femmes de Setné, dont l'action se passe dans l'Egypte ancienne. Si le cadre spatio-temporel d'un roman d'anticipation comme Les Navigateurs de l'infini se situe dans un avenir plus ou moins lointain ${ }^{25}$, le personnage principal reste néanmoins clairement identifiable comme un Européen. Le futur décrit offre, par ailleurs, suffisamment de similitude avec le présent du lecteur pour que celui-ci puisse s'y reconnaître. Dans les récits "préhistoriques ", qui excluent par définition la présence de l'homme moderne, le processus d'identification passe par la mise en évidence d'une relation généalogique, "raciale » ou simplement morale, avec le lecteur. Le personnage symbolisant le Même est ainsi parfois un individu appartenant à une population européenne archaïque, comme dans Vamireh ou Eyrimah. Dans un roman comme Le Félin géant, l'identité "raciale», rendue invraisemblable par la distance temporelle, est remplacée par une sorte de communauté idéologique. Aoûn, le protagoniste du récit, apparait en effet porteur d'un certain nombre de valeurs humanistes, tels l'amour ou la pitié, dans lesquelles le lecteur moderne peut se reconnaître. Ce qui suffit à en faire un représentant du Même. On pourrait appliquer la même remarque à un personnage exotique comme l'Egyptien Setné, qui est présenté, lui aussi, comme une sorte de 
précurseur lointain de l'humanisme moderne, notamment par sa conception idéaliste de la femme et de l'amour.

Face à ce premier personnage, l'autre élément du couple représente l'étrange ou le lointain. Son altérité apparaît complexe et multiple. Elle joue en effet sur plusieurs plans. Elle se situe d'abord sur un plan biologique : l'Autre appartient à une autre " race ", à une autre espèce, voire à une autre forme de vie. Mais l'altérité est aussi d'ordre culturel. L'Autre est celui qui possède une forme de pensée, un langage et une organisation sociale différents. L'altérité est encore, dans certains cas, de nature temporelle. L'Autre vit, réellement ou symboliquement, dans un autre temps que le présent du lecteur ou que celui du narrateur, qui en est la traduction dans le récit. Cette altérité temporelle est particulièrement importante dans les œuvres relevant du genre des "mondes perdus", qui mettent en scène des peuples « attardés ", témoins d'un stade antérieur de l'Histoire ou de l'Evolution humaine. La rencontre avec l'Autre apparait alors comme une sorte de voyage dans le passé, qui donne l'impression d'abolir le temps, même si l'on reste sur un plan symbolique, dans la mesure où ce n'est pas le passé en lui-même qui est retrouvé, mais une simple image de celui-ci ${ }^{26}$. Si cette forme d'altérité est généralement associée au passé, elle peut aussi être tournée vers le futur, comme dans Les Navigateurs de l'infini, où le narrateur et ses compagnons découvrent une planète qui leur apparaît comme l'image de l'avenir lointain de la Terre ${ }^{27}$. Inversant le schéma des récits de "mondes perdus", Rosny transforme le voyage interplanétaire accompli par ses personnages en un voyage vers l'avenir. Les Tripèdes martiens deviennent alors une sorte de représentation des hommes du futur, malgré la distance qui sépare les deux « espèces ». Une dernière forme d'altérité est constituée par la différence sexuelle, qui reste toujours présente, même lorsque l'Autre appartient à une espèce exotique, pratiquant un mode de reproduction totalement différent de celui des humains. Jacques Laverande, le narrateur des Navigateurs de l'infini, éprouve ainsi d'abord quelques difficultés à admettre que son attirance pour Grâce, la Tripède martienne, ait une origine sexuelle ${ }^{28}$. Il finit cependant par reconnaître que c'est bien « du désir » qu'il ressent « auprès d'elle à chaque frôlement de son corps" (LNI, p. 119).

Ces diverses formes d'altérité entrent dans des combinaisons variées, donnant à l'Autre une richesse et une profondeur qui en font tout à la fois le mystère et l'attrait. Le personnage de Grâce, dans Les Navigateurs de l'infini, réunit ainsi les quatre types d'altérité que nous avons distingués : elle appartient à une autre "espèce ", elle représente une autre forme de pensée et de culture, elle est située, symboliquement, dans un futur lointain ; elle incarne enfin, pour le narrateur, l'essence même de la féminité et de son mystère. Ce qui en fait une sorte d'Autre absolu, à l'étrangeté aussi déroutante que fascinante. Un récit de «mondes perdus » comme Le Trésor dans la neige propose une autre combinaison possible, qui rappelle la précédente, mais sur un mode mineur, dans la mesure où l'altérité biologique y est moins radicale. Les deux " épouses " préhistoriques du narrateur appartiennent en effet à la même espèce que lui, si elles ne sont pas de la même « race ». L'altérité temporelle est par ailleurs orientée différemment, vers le passé et non plus vers le futur. Celle-ci disparait, par contre, totalement dans les récits "préhistoriques ", où les acteurs principaux du récit, seuls concernés par la question du métissage, sont strictement contemporains les uns des autres, en termes d'évolution. Ils appartiennent par ailleurs tous à la même espèce, à défaut d'être de la même "race ", Rosny s'interdisant ici les audaces qu'il s'autorise ailleurs. De là, un certain affaiblissement de la différence entre le Même et l'Autre par rapport aux autres types de 
récit, notamment ceux qui relèvent du « merveilleux scientifique », où l'altérité de l'Autre prend une forme beaucoup plus radicale.

La simplicité de la formule : MEME + AUTRE se trouve encore compliquée par le fait que les personnages incarnant l'un ou l'autre des deux pôles sont parfois présentés comme des êtres ambigus, doués d'une double nature. Le Même et l'Autre apparaissent dès lors comme le résultat d'un mélange, qui vient brouiller la clarté de leur partage. Ce mélange peut résulter d'un premier métissage, antérieur à celui qui nous est rapporté dans le récit. C'est le cas de Glâva, dans Helgvor du fleuve bleu, qui est présentée comme le produit d'un premier croisement entre Tzohs et tribus autochtones: «Elle n'avait pas la tête cubique des Tzoh ni leurs sourcils obliques : une aïeule reparaissait dans son visage clair, dans les flammes rousses de ses yeux et dans son immense chevelure [...] Urm y reconnaissait la race des Lacs Verts, dont les guerriers Tzoh avaient jadis capturé des filles» (RP, p. 456). Cette double ascendance fait d'elle, par rapport à Helgvor, le jeune Ougmar qui la poursuit de son désir, une sorte de mélange de Même et d'Autre, dans la mesure où il retrouve en elle un certain nombre de traits de sa propre $"$ race $»^{29}$, préservés par un phénomène d'atavisme. La double nature du personnage prend, dans d'autres textes, un caractère plus étrange, comme dans La Jeune vampire, où le corps d'une jeune femme est possédé par l'esprit d'un être mystérieux, issu d'un autre "monde » ou d'une autre dimension. Durant le temps de sa possession, le personnage devient le lieu de rencontre de deux modes d'existence hétérogènes, l'un humain, l'autre non-humain, qui coexistent plus ou moins harmonieusement au sein d'un même organisme. On pourrait enfin citer le cas, plus ambigu, des Hommes-sangliers, qui, sans être à proprement parler des êtres doubles, n'en sont pas moins décrits comme le résultat d'une sorte de mélange fantastique entre l'homme et l'animal, voire entre différentes espèces animales. Rosny parle, à leur propos, «d'une étrange race animale, intermédiaire entre les grands singes et les sangliers» (RSF, p. 293). Comme le montrent ces différents exemples, c'est généralement le personnage incarnant l'Autre qui se trouve affecté d'une nature double. La situation inverse est plus rare. On pourra cependant citer ici le personnage principal de Dans le monde des Variants, Abel Faverol, qui est explicitement présenté comme un être double, à la fois humain et Variant, Même et Autre, ce qui lui permet de vivre simultanément dans chacun des deux univers ${ }^{30}$. L'origine de cette particularité reste inexpliquée, le lecteur devant l'accepter comme une donnée initiale, nécessaire au développement du récit.

Le métissage apparaît ainsi, chez Rosny, comme un phénomène complexe, aux formes multiples, même s'il reste fondamentalement placé sous le signe de la rencontre du Même et de l'Autre. Comme tel, il joue un rôle important, sinon central, dans ses récits, où il est présenté comme une alternative à la guerre et aux différentes entreprises de destruction qui constituent, pour Rosny, la loi générale de l'Histoire et de l'Evolution. S'il représente, dans un certain nombre de cas, le simple prolongement d'une relation d'alliance antérieure qui trouve là sa forme achevée, comme dans Le Félin géant ou Les Navigateurs de l'infini, il apparaît ailleurs comme un signe de réconciliation entre peuples ennemis, dont il est censé garantir la coexistence pacifique. Un roman comme Eyrimah est exemplaire, à cet égard, dans la mesure où il montre comment deux " races ", d'abord engagées dans une lutte mortelle et stérile, finissent par renoncer à s'affronter pour unir leur destin, à travers la constitution de deux couples mixtes, qui annonce leur prochaine « fusion » (RP, p. 194). Rosny parle, à ce propos, de « l'éternel mystère qui vainc la Destruction » (ibid., RP , p. 201). A la violence aveugle et destructrice, le métissage substitue le désir et l'amour, 
gage de paix et de concorde.. Cela ne signifie pas que le métissage soit exempt de tout élément de violence. De fait, un certain nombre de couples « mixtes » sont le résultat d'un acte de violence initial, dont la femme est généralement la victime : rapt (Vamireh, Elem d'Asie, Nymphée, L'Etonnant voyage de Hareton Ironcastle), viol (Les Hommes-sangliers, La Sauvage aventure), capture (Eyrimah). Cet acte joue, dans certains cas, le rôle d'élément déclencheur des hostilités entre les peuples, comme dans Vamireh, où le rapt d'Elem est à l'origine du premier affrontement entre "Européens" et "Asiatiques ». La violence trouve ici son origine dans le Désir, souvent présenté par Rosny comme un instinct primitif de prédation. Or le désir prend, dans ses récits, une forme d'autant plus impérieuse qu'il vise un être "autre». Comme l'écrit A. Gourdet, "Chez Rosny, l'hétérogène est érogène $»^{31}$. La rencontre avec l'Autre est, en ce sens, particulièrement favorable à l'exacerbation des instincts élémentaires. Si cet Autre appartient, de surcroît, à une "race" ennemie, le désir sensuel se double d'une obscure volonté d'humilier l'adversaire, à travers le traitement infligé à sa composante féminine. Autant d'éléments qui créent les conditions d'une relation ambiguë à la violence. Le métissage n'en reste pas moins une alternative à celle-ci, dans la mesure où il est amené à dépasser sa propre origine, par une sorte de processus de sublimation, qui conduit du désir prédateur à l'amour et au respect de l'autre. Comme l'écrit Rosny, dans un autre contexte, "l'amour continue [ici] étrangement la guerre " ${ }^{32}$. C'est, par exemple, le chemin suivi par Vamireh, qui évolue du statut de ravisseur à celui d'amant protecteur. Cette métamorphose suppose cependant un certain laps de temps, nécessaire à une meilleure compréhension mutuelle, ne serait-ce qu'à travers l'apprentissage de la langue de l'autre. En l'absence de ce délai, les relations au sein du couple resteront marquées par la violence, comme dans Les Hommes-sangliers, où Suzanne Dejongh et son ravisseur n'ont pas la possibilité de dépasser ce stade préliminaire, malgré une certaine évolution de leurs rapports au cours du récit.

Le métissage s'inscrit par-là dans une vision générale de l'Histoire et de l'Evolution, conçue comme le lieu d'affrontement de forces antagonistes, qui sont celles de la Vie et de la Mort. Un passage d'Eyrimah évoque, sur un mode lyrique, « la guerre » qui « s'agite à côté du poème d'amour ", mais lui est en même temps «si étroitement liée » « dans la nature » (RP, p. 178-179). Le métissage est clairement présenté chez Rosny comme étant du côté de la Vie. Il participe de cette force, présente dans l'ensemble de l'univers, qui lutte, souvent victorieusement, contre «la Destruction» (ibid., RP, p. 201). De là, son association fréquente, chez Rosny, avec l'idée de régénération. Il s'agira parfois simplement de sauver un peuple menacé d'anéantissement, en lui assurant une descendance. Aoûn arrache ainsi, dans Le Félin géant, la horde des Louves à une disparition certaine en leur apportant l'élément mâle qui leur fait défaut, les hommes de leur tribu ayant tous été massacrés. Le métissage joue le même rôle dans un certain nombre de récits de "mondes perdus », où l'on nous présente de petits groupes d'humains survivant tant bien que mal dans un milieu à l'équilibre fragile. Pour ces survivants, souvent menacés d'extinction, le métissage avec les Européens représente la seule possibilité de survie. C'est le cas dans Le Trésor dans la neige, où la tribu préhistorique rencontrée par Alglave, le personnage principal, ne compte plus qu'un homme et deux femmes en état de procréer. Sa venue et les «mariages » successifs qui s'ensuivent permettent de relancer le processus de reproduction et d'assurer un avenir à la tribu. La situation apparait plus critique encore dans La Résurrection de mon oncle Jérôme, où le «monde perdu » découvert par le narrateur n'est habité que par deux personnes, une femme et son fils. La survie du groupe passe ici encore nécessairement par le métissage. 
17 Dans d'autres cas, le processus de régénération prend la forme plus complexe d'une restitution de l'énergie vitale. Il s'agit alors de lutter, par le métissage, contre le phénomène de déclin, souvent accompagné de dégénérescence, qui frappe les "races » trop anciennes. Ce phénomène, souvent évoqué par Rosny, qui partage avec ses contemporains la hantise de la décadence ${ }^{33}$, reçoit différentes explications, qui se ramènent toutes à l'idée d'une sorte d'épuisement progressif de l'énergie vitale de la « race ", dont le destin suit la même courbe ascendante, puis descendante, que la vie de l'individu. Rosny donne un tableau saisissant d'un de ces peuples «décadents » dans le passage, souvent cité, de La Guerre $d u$ feu, où il évoque le destin des Hommes-sansEpaules : «[...] sans qu'ils eussent subi d'autres cataclysmes que les autres hommes, leur croissance s'arrêta. [..] Leurs corps devinrent plus étroits et plus lents ; leur langage cessa de s'enrichir, puis il s'appauvrit [...] Vite las, ils mangeaient peu et dormaient beaucoup $[. .$.$] « (R P$, p. 307). Ce processus de déclin est présenté comme à peu près irréversible, une fois enclenché. Le seul espoir pour les peuples décadents réside dans la possibilité d'unir leur destin biologique à une « race " plus jeune et plus vigoureuse, qui leur transmette sa vitalité, leur permettant par-là même d'échapper à l'extinction. C'est le cas dans Eyrimah, où les Montagnards, héritiers des vieilles civilisations magdaléniennes déclinantes, trouvent dans le métissage avec la race plus dynamique des envahisseurs asiatiques une nouvelle vitalité, qui leur redonne une place dans l'Histoire. Les Navigateurs de l'infini constituent une autre variation sur ce thème. Explicitement présentés comme des vaincus de l'Histoire, des "décadents ${ }^{34}$, les Tripèdes retrouvent au contact des Terriens une certaine énergie, qui les arrache à leur inertie et à leur fatalisme. La naissance de l'enfant de Grâce et de Jacques Laverande, à la fin du récit, est le signe le plus spectaculaire de cette renaissance, même s'il s'agit là d'un événement destiné à rester sans lendemain, comme le suggère clairement le texte, qui exclut la possibilité d'une fusion des deux espèces. Le même processus de rajeunissement est à l'œuvre dans les récits de « mondes perdus » où des Européens, héritiers de longs siècles de civilisation, se trouvent confrontés à des peuples primitifs ayant conservé toute la fraîcheur et l'énergie de la «jeunesse du monde $»^{35}$. En unissant leur destin à celle de ces peuples, les Européens peuvent espérer capter cette énergie à leur profit et écarter ainsi la menace de déclin qui menace, selon Rosny, toutes les vieilles civilisations ${ }^{36}$. Ils apportent en contrepartie, nous l'avons vu, l'espoir d'une survie à des peuples et des cultures condamnés à disparaître, chacun des deux partenaires trouvant ainsi dans son union avec l'autre un avantage décisif. Il est vrai que cette opération ne va pas non plus sans danger pour les uns et les autres. Pour les habitants des «mondes perdus", l'arrivée des Européens coïncide souvent avec la destruction de leur milieu, comme dans Le Trésor dans la neige ${ }^{37}$. Le retour aux origines comporte, de même, certains risques pour les Européens, dont il fragilise le délicat équilibre psychologique et moral. C'est l'expérience tragique vécue par Suzanne Dejongh dans Les Hommes-sangliers. Incapable d'affronter ses réactions ambivalentes face à un monde primitif, dont la violence lui est brutalement révélée, elle choisit le suicide et la mort.

18 A cette dimension biologique du métissage, essentielle pour Rosny, qui retrouve ainsi certaines préoccupations eugénistes de ses contemporains, s'ajoute une dimension culturelle, tout aussi fondamentale. La «fusion des races" s'accompagne en effet nécessairement d'un mélange des cultures, à travers un processus d'apprentissage et d'échange, qui commence par l'apprentissage de la langue de l'autre. Rosny évoque souvent, dans ses récits, ce moment des premiers échanges, encore maladroits, où chacun 
s'efforce de comprendre les mots ou les signes de son partenaire ${ }^{38}$. Moment privilégié où s'établit un début de communication entre les personnages, qui cessent par-là même d'être des étrangers l'un pour l'autre. A partir de cette ouverture initiale, un véritable dialogue va pouvoir se développer, qui permettra de faire circuler un flux croissant d'informations. Se crée ainsi une dynamique qui répond, comme le montre Rosny, à une profonde curiosité pour le monde de l'autre, notamment de la part des personnages masculins, fascinés par une altérité aussi troublante qu'irritante dans sa différence énigmatique. Cette volonté de savoir prolonge l'élan du désir, qui trouve là une forme d'expression plus abstraite et moins violente que la simple possession physique, Rosny soulignant, ici comme ailleurs, la capacité du désir à évoluer vers des formes plus civilisées. Le métissage apparaît ainsi comme un facteur de progrès pour l'Humanité, par les contacts qu'il permet entre les cultures. Ces contacts peuvent, il est vrai, se développer en-dehors du processus de métissage biologique. C'est, par exemple, le cas dans La Guerre du feu, où Noah acquiert auprès des Homme-sans-épaules un certain nombre de techniques, sans qu'il y ait de mélange des «races ». Il en va, de même, dans Les Navigateurs de l'infini, avec les Ethéraux, dont les relations avec les Terriens restent purement intellectuelles. Le métissage biologique n'en reste pas moins, chez Rosny, la voie privilégiée par laquelle passent les échanges culturels, et par-là le progrès de l'Humanité, qu'il soit d'ordre intellectuel ou moral.

Le mélange des « races » et des espèces conduit, en effet, à dépasser les limites étroites de l'identité "raciale", pour s'ouvrir à une vie morale plus large, où l'Autre se voit reconnaître, par-delà ses particularités biologiques et culturelles, la qualité générale de semblable. Ce qui entraîne l'apparition de toute une série de sentiments altruistes, comme l'amour ou la pitié, dont les récits "préhistoriques » de Rosny nous décrivent l'émergence progressive dans un monde encore largement dominé par la férocité des instincts élémentaires. Le métissage se voit ainsi attribuer un rôle important dans le processus évolutif qui a mené l'homme de l'état de brute soumise au jeu aveugle de ses pulsions à celui d'être civilisé. Ce processus est cependant loin d'être achevé pour Rosny, qui appelle à un nouvel élargissement des sentiments altruistes au-delà des frontières de l'espèce humaine. Toutes les formes de vie intelligente, où qu'elles se trouvent dans l'univers, doivent être reconnues comme des semblables, dignes comme tels de respect, malgré leur étrangeté parfois inquiétante ${ }^{39}$. Dans cet élargissement de la conscience morale aux dimensions du cosmos, qui doit faire de l'homme le «compagnon de l'univers ", pour reprendre le titre de l'un des derniers ouvrages de Rosny, le métissage conserve toute son importance, comme le montre un récit comme Les Navigateurs de l'infini ou La Jeune vampire. Dans un cas comme dans l'autre, c'est à travers le lien créé par le désir que s'instaure une relation pacifique avec l'Autre, fondée sur la reconnaissance de sa dignité et le respect de ses droits ${ }^{40}$.

Expression des forces profondes de la Vie en lutte contre l'instinct de Mort, vecteur de progrès intellectuel et moral, le métissage fait ainsi l'objet, chez Rosny, d'une sorte de célébration "mystique ", qui associe rêveries panthéistiques et engagement humaniste dans une synthèse généreuse et visionnaire, caractéristique du positivisme « lyrique » de l'auteur. Les récits "conjecturaux» de Rosny peuvent être lus, en ce sens, comme un plaidoyer en faveur du " mélange des races » et de l'ouverture à l'Autre. Une lecture plus attentive des œuvres révèle cependant chez lui une attitude pour le moins ambivalente vis-à-vis d'un processus qui engage l'avenir biologique et culturel des peuples. Cette ambivalence est perceptible dans le traitement narratif du thème lui-même, qui tend à 
limiter la portée des thèses défendues par Rosny, quand il n'en modifie pas sensiblement la signification, les textes montrant d'une certain façon autre chose que ce qu'ils disent ${ }^{41}$. On peut ainsi souligner le fait que, s'il est souvent question de couples «mixtes » et de «mélange des races » dans les récits de Rosny, le processus de métissage est, par contre, rarement représenté dans sa réalité concrète. Il est significatif, de ce point de vue, que, sur les seize textes que nous avons retenus, seuls cinq ${ }^{42}$ évoquent la naissance d'un enfant métis, concrétisant la fusion de deux « races » ou de deux espèces. On trouve, de même, très peu de métis parmi les personnages, qui appartiennent presque toujours à une race " pure ». Glâva et sa sœur Amhao, les deux héroïnes d'Helgvor du fleuve bleu, constituent l'une des rares exceptions, sinon la seule, à cette règle générale ${ }^{43}$. Et encore leur statut de métis est-il plus ou moins occulté par le texte. Rosny semble en effet vouloir faire oublier l'origine "mêlée » de ses personnages, en attribuant à chacune des deux jeunes femmes des caractéristiques permettant de les rattacher à un type « racial » défini, conservé dans sa pureté par les hasards de l'hérédité. L'une des sœurs présente ainsi tous les traits des Tzoh asiatiques, leur «visage opaque, leurs mâchoires d'aurochs, leurs yeux de corneilles », tandis que l'autre correspond au type européen, avec « son visage [...] étroit, ses yeux clairs, ses cheveux de lionne et son torse flexible» (RP, p. 483). Tout se passe comme si la logique de la "pureté raciale » l'emportait ici sur la réalité du métissage, qui n'est mentionné que pour être aussitôt refoulé par le texte ${ }^{44}$.

21 En-dehors de ces quelques exemples, le métissage reste au mieux une simple virtualité, dont la réalisation est renvoyée à un avenir plus ou moins lointain, échappant comme tel à l'espace du récit. C'est notamment le cas dans les fictions "préhistoriques", qui se concluent généralement par la promesse d'une "fusion des races ", devant ouvrir une ère de paix et de progrès pour l'espèce humaine. Mais cette promesse, reconduite de texte en texte, ne se concrétise jamais, malgré la présence ponctuelle de quelques rares individus métis, comme dans Helgvor du fleuve bleu. Les peuples, enfermés dans leur identité " raciale ", semblent dès lors condamnés à s'affronter dans une guerre éternelle, dont nous suivons le développement, à travers l'ensemble du cycle, depuis les temps lointains " où l'homme ne traçait encore aucune figure sur la pierre ni sur la corne » (RP, p. 205) jusqu'aux premières civilisations de l'âge du bronze. De là, une impression de ressassement de l'Histoire, vouée à se répéter sans fin, qui contraste avec l'image du Progrès, développée par ailleurs dans les romans "préhistoriques" de Rosny, censés décrire la lente ascension de l'Humanité vers une existence moins soumise à la loi brutale de l'Instinct et plus consciente d'elle-même ${ }^{45}$. L'impression de répétition est particulièrement sensible dans les trois romans (Vamireh, Eyrimah, Helgvor du fleuve bleu) consacrés au conflit qui aurait opposé, à partir de la fin du Paléolithique, des Orientaux aux populations européennes autochtones ${ }^{46}$. D'un texte à l'autre, nous assistons au même déchaînement de violence entre les deux « races" ennemies, généralement suivi d'une réconciliation, sanctionnée par la perspective d'une prochaine "fusion des races", qui est régulièrement oubliée dans le volume suivant, où le même cycle de haine et de violence se déroule à nouveau. La seule évolution perceptible concerne le degré d'intensité du conflit, qui se radicalise un peu plus à chaque fois, prenant progressivement les dimensions d'une guerre totale, aux visées génocidaires. Dans le dernier roman de la série, on ne trouve même pas l'habituelle réconciliation finale, les Tzoh asiatiques étant apparemment massacrés jusqu'au dernier par les Ougmar et leurs alliés ${ }^{47}$. La perspective d'une « fusion des races ", sans cesse différée, tend ainsi à s'effacer devant la réalité d'une relation purement conflictuelle, de plus en plus violente, dont la seule issue concevable semble être l'extermination de l'un des deux adversaires. Rosny évoque bien au début d' 
Eyrimah, dans le tableau général qui ouvre le récit, l'apparition d'une "race mixte ", "bientôt dominante» (RP, p.101), dans laquelle le lecteur est invité à reconnaître l'origine lointaine des populations européennes modernes. Mais ce fait historique, ou considéré comme tel par les préhistoriens de la fin du XIXe siècle, ne joue aucun rôle sur le plan narratif, que ce soit dans le roman lui-même ou dans l'ensemble du cycle. A peine mentionné, il est comme mis entre parenthèses par le récit, qui revient, une fois de plus, sur le thème du «duel des races $»^{48}$, déjà traité dans Vamireh et qui sera de nouveau développé dans Helgvor du fleuve bleu.

Dans un certain nombre de textes de notre corpus, c'est la possibilité même du métissage qui se trouve exclue par le récit. Toute perspective, même lointaine, d'une « fusion des races » disparaît alors, les couples « mixtes » concernés étant condamnés à rester sans descendance. Cet échec du processus de métissage reçoit différentes justifications sur le plan narratif. Il est ainsi parfois expliqué par l'absence de relations physiques entre les deux éléments du couple, qui demeure ainsi purement platonique, cette situation se trouvant elle-même justifiée par la brièveté de la période de cohabitation entre les deux individus concernés et les aléas d'une intrigue souvent riche en péripéties, qui laisse peu de temps aux personnages pour assouvir leurs désirs. Plusieurs récits, parmi ceux que nous avons retenus, recourent à cet artifice narratif, qui souffre d'une certaine invraisemblance, même s'il est conforme aux conventions du roman d'aventure de la fin du XIXe siècle. Dans Nymphée, la jeune européenne enlevée par les Hommes-des-eaux échappe ainsi à la souillure d'un viol grâce à l'intervention providentielle de son " fiancé » et de ses alliés, qui l'arrachent à ses ravisseurs avant que l'irréparable ne se soit produit. Le même schéma narratif est repris dans L'Etonnant voyage de Hareton Ironcastle. On y voit une fois encore une jeune femme - Muriel, la fille de Hareton Ironcastle, prisonnière d'une tribu d'hommes primitifs, les Hommes-Trapus, sauvée d'un sort funeste par l'action de ses compagnons d'aventure. Confrontée à une situation similaire, bien que plus dramatique encore, puisqu'au risque de viol s'ajoute la perspective d'une mort atroce, Glâva, l'héroïne d'Helgvor du fleuve bleu devra, quant à elle, son salut à l'attaque imprévue d'une tribu ennemie, qui lui permettra de s'enfuir et de se soustraire, par la même occasion, aux désirs du chef de ses ravisseurs ${ }^{49}$. Dans d'autres textes, le personnage féminin trouve une mort prématurée, avant que se dessine la perspective d'un éventuel métissage, comme dans Les Hommes-sangliers. Par son suicide, Suzanne Dejongh dénoue un conflit intérieur psychologique et moral insoluble, tout en coupant court brutalement à toute possibilité d'une " fusion des races ». La Fille bleue propose une autre variation sur le même thème. La mort du personnage féminin est ici provoquée par l'attitude du narrateur, qui, par « respect de la personnalité féminine » (LFB, p. 277), refuse de s'unir à la jeune vierge qui lui est offerte par la tribu des Hommes Bleus. Son comportement est interprété par les indigènes comme un signe de mécontentement à l'égard de sa partenaire, qu'ils s'empressent d'exécuter. La jeune indigène meurt ainsi victime de lois ancestrales aveugles et des préjugés moraux du narrateur, qui n'a pas su s'adapter à un nouvel environnement culturel, obéissant à d'autres règles que celles qui ont cours dans son propre monde.

Il peut enfin arriver que le métissage soit rendu impossible ou improbable par la distance biologique qui sépare les deux "races» ou les deux espèces concernées, comme dans $L a$ Sauvage aventure, où la jeune européenne enlevée et violée par le chef des HommesKérabaus doit apparemment à cet heureux hasard d'échapper à la "souillure " d'un métissage déshonorant, qui aurait définitivement compromis ses chances de retrouver sa 
place dans la société. Un passage du roman suggère, il est vrai, que les Hommes-Kérabaus pourraient "se reproduire avec d'autres humains" "à la manière limitée dont les onagres se reproduisent avec les chevaux " (LSA, p. 104). Mais cette hypothèse n'est pas confirmée par la suite du récit, qui insiste, au contraire, fortement sur l'écart entre les deux "espèces ", issues de lignées différentes. Comme dans les exemples précédents, l'impossibilité matérielle du métissage apparait ici comme l'expression plus ou moins transparente d'un véritable interdit moral, pesant sur les relations entre individus de « races » ou d'espèces différentes. Il n'est pas indifférent que la plupart des textes que nous avons cités mettent en scène des couples mixtes composés d'une Européenne et d'un individu appartenant à un peuple "primitif » ou à une race "inférieure ", La Fille bleue constituant la seule exception. Le métissage, voire la simple existence de relations sexuelles, apparaît dans ce contexte comme une transgression majeure, à la fois insupportable et impensable, que le récit s'emploie à conjurer par différents artifices narratifs. Encore le viol est-il parfois consommé, alors que nous n'avons aucun exemple de croisement entre une Européenne et un homme "primitif ». Comme s'il y avait là un interdit plus absolu encore que celui pesant sur la sexualité féminine.

Dans ses détours et ses distorsions, l'œuvre "conjecturale" de Rosny laisse ainsi clairement apparaitre une véritable résistance à l'idée de métissage, malgré le discours de tolérance et d'ouverture, par ailleurs affiché. Cette résistance est perceptible là même où le métissage, cessant d'être une réalité purement virtuelle ou interdite, acquiert une présence concrète sur le plan narratif, avec l'apparition d'enfants métis ou de personnages d'origine "mêlée ». Il est en effet frappant de constater que, dans ce cas, le processus de "fusion des races ", censé associer le Même et l'Autre, se transforme en simple reproduction du Même ou de l'Autre, chaque élément se trouvant conservé dans son état de "pureté » initial. A la synthèse promise se substitue ainsi une sorte de processus analytique, qui maintient séparé ce qui devrait être uni. L'exemple le plus net de ce phénomène est offert par Glâva et Amhao, les deux hérö̈nes d'Helgvor du fleuve bleu. Théoriquement issues du croisement d'individus appartenant à deux « races » différentes, elles sont décrites, nous l'avons vu, comme incarnant chacune un type « racial» pur, comme si les deux éléments initiaux ne réussissaient pas à s'unir dans un type synthétique ${ }^{50}$. Le métissage reste dès lors purement théorique, la "fusion des races" promise n'ayant pas eu lieu. L'intrigue amoureuse qui structure le roman prend, corrélativement, une signification particulière, très différente de celle que l'on peut observer dans les autres fictions préhistoriques, où le désir érotique est généralement l'expression d'une fascination pour l'Autre en tant que tel. Le désir naît ici inversement d'un sentiment de familiarité, d'une reconnaissance du Même par le Même, d'autant plus inattendue que celui-ci apparaît chez l'Autre. La réaction d'Helgvor, lors de sa première rencontre avec l'héroïne est révélatrice, à cet égard. S'il est rebuté par l'apparence d'Amhao, qui « montr[e] le visage opaque des Tzoh, leurs mâchoires d'aurochs, leurs yeux de corneilles", il est au contraire immédiatement attiré par sa sœur "qui ressembl[e] étrangement aux femmes Ougmar » $(R P, \mathrm{p} .483)$. L'étrangeté, c'est précisément de retrouver le Même chez l'Autre. La relation exogamique se révèle ainsi paradoxalement endogamique: si elle appartient en effet culturellement au peuple des Tzoh, Glâva est biologiquement de la même «race » qu'Helgvor ${ }^{51}$. En s'unissant avec lui, elle ne fait que retrouver une origine obscurément recherchée dans une sorte d'odyssée symbolique qui efface le drame oublié de l'aïeule arrachée à sa tribu et contrainte de vivre avec l'ennemi détesté52. 
25 La plupart des enfants métis qui apparaissent dans les textes de notre corpus sont marqués par le même phénomène d'hérédité unilatérale, qui fait d'eux la réplique presque parfaite de l'un de leurs deux parents, le plus souvent celui censé représenter le pôle de l'Altérité. Abel, le narrateur-protagoniste d'Un Autre monde, engendre ainsi un fils qui est décrit comme la reproduction exacte de son père, dont il présente les mêmes caractéristiques singulières : «[...] un enfant nous est né, et cet enfant réunit toutes les caractéristiques de ma constitution. Couleur, vision, rapidité extrême de mouvement, nutrition, il promet d'être l'exacte réédition de mon organisme " (RSF, p. 46-47. C'est nous qui soulignons). L'enfant de Grâce apparaît, de même, comme un pur Martien, même s'il est voué par sa mère «à la Terre, sa patrie» (LNI, p. 251). Son appartenance à l'espèce martienne est affirmée sans équivoque par sa mère : «C'est un Martien ... je l'ai voulu ainsi! «(Ibid.). Cette déclaration prend dans le contexte du roman un sens précis, dans la mesure où Rosny attribue aux Martiennes la possibilité de se reproduire, si elles le souhaitent, par une sorte de processus de parthénogenèse, excluant toute forme de fécondation externe ${ }^{53}$. C'est ce mode de reproduction qu'a choisi la jeune Martienne de préférence à un mode de reproduction plus classique ${ }^{54}$, qui était apparemment possible malgré la distance considérable entre les deux espèces, Martienne et Terrienne. On pourrait encore citer le fils de la "jeune vampire », présenté lui aussi comme la réplique de sa mère, dont il a hérité la même pâleur et le même mode de nutrition singulier, même s'il semble plus « inoffensif », selon l'expression du narrateur ${ }^{55}$. Le cas de l'enfant métis de Dans le monde des variants apparaît plus ambigu, dans la mesure où il ne ressemble pas totalement à sa mère, Liliale, même s'il appartient clairement au monde des Variants, ayant perdu la faculté de vivre « doublement » (RSF, p. 112) dans deux univers différents comme son père. Il possède en effet « un sens du monde humain qui manqu[e] à sa mère » (ibid.), et qui est peut-être un héritage paternel, bien que le texte ne donne aucune indication précise sur ce point. L'interprétation de son cas est encore compliquée par le fait que le couple mixte dont il est issu compte un élément double, mélange de Même et d'Autre. La ressemblance de l'enfant avec sa mère peut dès lors être interprétée de deux façons différentes, soit comme un nouvel exemple d'hérédité unilatérale, soit comme le résultat d'un processus de fusion de l'héritage biologique maternel et d'une partie de l'héritage biologique paternel, préalablement dissocié en ses deux composantes, celle relevant du Même et celle relevant de l'Autre ${ }^{56}$. Dans un cas, il n'y a pas réellement de métissage, l'Autre ne faisant que se reproduire à l'identique; dans l'autre, il y a bien métissage, mais le mélange ainsi obtenu est homogène et, comme tel, indiscernable d'un produit biologiquement " pur ». Quelle que soit l'hypothèse retenue, on aboutit à la même représentation paradoxale d'un métissage qui conserve les identités biologiques ou raciales, au lieu de les dissoudre.

Le Trésor dans la neige est le seul texte de notre corpus qui mette en scène un enfant authentiquement métis, échappant comme tel au phénomène d'hérédité unilatérale. Il s'agit de la fille d'Alglave et de Namhâ, dont le texte de Rosny souligne de façon insistante le double héritage "racial : «[...] Namhâ donn[a] le jour à une fille dans les veines de laquelle coulait une part de sang moderne" (RSF, p. 344). Ce personnage ne joue cependant qu'un rôle très secondaire dans le récit, où elle n'apparaît que de façon très ponctuelle ${ }^{57}$. Elle n'est même pas mentionnée dans l'épilogue, où s'impose au contraire la figure de Rahouam, le fils d'Awah et de Touanhô, décrit par le narrateur comme « de pure race préhistorique » (ibid.). Avec lui, c'est le métissage culturel qui se trouve mis en avant, au détriment du métissage biologique, plus ou moins marginalisé. Le fils d'Awah est en 
effet présenté comme le produit d'un processus d'acculturation particulièrement réussi, qui a fait de lui un artiste exceptionnel, associant « le génie statuaire des Préhistoriques » à la « discipline » savante des Modernes (RSF, p. 363). Le métissage culturel tend ainsi à se substituer au métissage biologique comme forme de relation privilégiée entre les « races » et les peuples. Cette substitution est symbolisée par la relation quasi paternelle qu'entretient Alglave avec le jeune homme, dont il semble beaucoup plus proche affectivement et intellectuellement que de ses propres enfants, rapidement évoqués au détour d'une phrase ${ }^{58}$. Le métissage culturel a l'intérêt d'être compatible avec le maintien d'une "race pure", représentée ici par la descendance d'Awah, qui forme une lignée parallèle à celle d'Alglave. La survie de "l'antique race tourassienne" (RSF, p. 363) constitue en effet un enjeu majeur du texte, qui propose une variation originale sur le topos classique de la destruction du «monde perdu». De fait, grâce à Awah et à ses nombreux enfants, l'avenir de la tribu préhistorique paraît assuré, comme le souligne Alglave $^{59}$. La survie du groupe n'aurait cependant qu'un intérêt limité si la pureté de la « race » n'était pas préservée d'une façon ou d'une autre. Ce qui suppose que subsiste un certain nombre d'individus échappant au processus de métissage biologique, qui se voit ainsi limité dans son extension. La coexistence de deux lignées, l'une "pure ", l'autre métissée, apparaît en ce sens comme un compromis entre la nécessité d'un brassage "génétique ", pour utiliser une expression anachronique, et la préservation tout aussi nécessaire de l'identité raciale.

Ce thème de la double lignée se retrouve dans d'autres textes de notre corpus, notamment dans Les Navigateurs de l'infini et dans La Jeune vampire, où Rosny imagine des situations sentimentales complexes, fondées sur la double relation affective, simultanée ou successive, d'un homme et de deux "femmes", dont l'une appartient, comme son partenaire masculin, à l'espèce humaine, tandis que l'autre vient d'ailleurs ${ }^{60}$. Cette relation triangulaire débouche, chaque fois, sur la création de deux lignées parallèles, présentant des caractéristiques «raciales» ou biologiques nettement différenciées. Jacques Laverande a ainsi un enfant de chacune de ses deux "épouses", Grâce la Martienne et Violaine la Terrienne, dont la maternité simultanée accentue encore l'effet de parallélisme recherché par le texte ${ }^{61}$. La même relation triangulaire apparaît dans $L a$ Jeune vampire, même si la situation se trouve compliquée ici par l'indistinction physique et sociale des deux «femmes » qui partagent le même corps et le même nom. D’abord marié à une mystérieuse entité féminine venue d'un autre univers, James Bluewinkle vit ensuite avec la véritable Evelyn Grovedale, qui a entre temps réintégré son corps. Du premier « mariage » naît Walter Bluewinkle, l'enfant-vampire, du second ses demi-frères humains, mentionnés au début du récit ${ }^{62}$. L'opposition entre lignée métisse et lignée de «race pure " tend cependant à se confondre ici avec une simple différence de "race» ou d'espèce, dans la mesure où le processus de métissage se réduit plus ou moins dans les deux textes à une simple reproduction de l'Autre, comme nous l'avons montré précédemment. La coexistence de deux lignées prend dès lors un sens sensiblement différent de celui qu'elle avait dans Le Trésor dans la neige. Il s'agit moins de réaffirmer, à travers cet artifice narratif, la nécessité de préserver une forme de pureté « raciale » que de souligner le destin parallèle des "races» ou des espèces concernées, condamnées à vivre l'une à côté de l'autre, sans pouvoir réellement se mélanger, la logique de l'identité s'imposant une fois encore au détriment de la fusion des « races » ou des espèces. faite à la fois d'exaltation lyrique et de résistance plus ou moins inconsciente, qui 
s'exprime par une sorte de double discours dans le récit. Ce qui conduit à nuancer quelque peu l'image, valorisée par la critique actuelle, d'un auteur adepte d'un métissage généralisé et d'une ouverture totale à l'Autre. La lecture de ses textes fait apparaitre une tension entre un humanisme universaliste, élargi à toutes les formes de vie intelligente, quelles qu'elles soient, appelées à former une vaste communauté intellectuelle et morale, par-delà les frontières biologiques et culturelles, et une vision du monde racialiste, commune à la plupart des contemporains de Rosny, qui postule l'existence de " races » profondément différentes les unes des autres et hiérarchisées en elles. La présence de cet arrière-plan racialiste est clairement perceptible dans des récits comme La Sauvage aventure ou Les Hommes sangliers, où le rapprochement brutal entre « races inférieures » et «races supérieures» prend la forme dramatique d'un événement traumatisant, générateur d'angoisse et de mort pour les personnages. De façon plus générale, on peut voir dans l'attitude ambiguë de Rosny vis-à-vis du métissage l'expression d'une relation profondément ambivalente à l'Autre. Si l'Altérité est chez lui source de fascination et de désir, elle n'en reste pas moins fondamentalement inquiétante, quand elle ne suscite pas la peur ou la répulsion. Les textes de notre corpus contiennent ainsi de nombreux exemples de figures négatives de l'Autre, du primitif proche de l'animalité par ses instincts violents et sa sexualité agressive (L'Etonnant voyage de Hareton Ironcastle, La Sauvage aventure, Les Hommes-sangliers) à la femme-vampire, qui absorbe la force vitale de son entourage (La Jeune vampire), en passant par le barbare sanguinaire, fanatisé par une religion homicide (Eyrimah, Helgvor du fleuve bleu). Même des êtres aussi paisibles en apparence que les Tripèdes martiens finissent par se révéler inquiétants et l'épilogue des Navigateurs de l'infini laisse planer un doute sur l'avenir des relations entre les deux espèces, apparemment reprises dans le jeu de la concurrence vitale ${ }^{63}$.

Au-delà de cette dimension idéologique et fantasmatique, la question du métissage apparait étroitement liée chez Rosny à une réflexion sur le Temps et l'Histoire. La « fusion des races » constitue en effet, pour lui, une loi générale de l'Histoire de l'Humanité, dont le développement s'accompagne d'une ouverture progressive des différents groupes humains les uns aux autres. Si ce processus représente un facteur de progrès essentiel sur le plan intellectuel et moral, comme le souligne Rosny, qui s'oppose par là clairement aux philosophies de l'Histoire associant métissage et décadence ${ }^{64}$, il entraîne cependant un certain appauvrissement de la diversité biologique et culturelle de l'espèce humaine, aboutissant à un effacement progressif de l'Autre en tant que tel. Ce qui ne peut manquer de susciter une réaction négative chez un écrivain et un penseur qui a placé l'ensemble de son œuvre sous le signe d'un pluralisme "intégral», privilégiant une ontologie du multiple. L'attitude prêtée à Alglave, dans la conclusion du Trésor dans la neige, est significative à cet égard. Craignant que son "rêve » de retour aux origines soit «moins captivant » si sa compagne parlait "une autre langue que la sienne» (RSF, p. 363), il renonce à lui enseigner le Français ou toute autre langue moderne. C'est une façon pour lui de maintenir une distance, en refusant de mener à son terme un processus d'acculturation, déjà partiellement engagée $e^{65}$, qui risque de rendre les deux personnages trop proches l'un de l'autre et de priver par là même la jeune femme de son charme « mystérieux » et « lointain » (ibid.). Il s'agit de préserver par là l'altérité de l'Autre d'une assimilation destructrice. Rosny parle ici, il est vrai, de métissage culturel et non de métissage biologique. L'attitude du personnage n'en est pas moins révélatrice d'une interrogation inquiète devant un phénomène dont la signification reste finalement ambiguë. Cette interrogation s'inscrit chez Rosny dans le cadre plus large d'une réflexion critique sur l'Histoire, perçue à la fois comme une lente évolution vers des formes 
d'existence intellectuelle et morale toujours plus complexes et un processus entropique conduisant à l'épuisement progressif de l'énergie créatrice de l'Humanitée ${ }^{66}$.

Relus dans cette perspective, les textes de notre corpus peuvent apparaître comme une tentative symbolique pour neutraliser par l'Ecriture les conséquences du métissage, le double discours tenu dans ces récits permettant de maintenir la nécessité « morale » d'un Progrès par ailleurs inéluctable, dont le processus de "fusion des races " constitue un élément essentiel, tout en préservant une diversité anthropologique menacée par ce même processus. Rosny construit par là une sorte d'espace utopique, où l'action destructrice du Temps et de l'Histoire se trouve provisoirement suspendue. La pratique de l'hybridation générique, particulièrement présente dans les textes "conjecturaux ", qui appartiennent souvent à plusieurs genres à la fois ${ }^{67}$, participe de la même utopie, dans la mesure où elle représente une version euphorique du métissage, ramené à un pur jeu formel, où l'imagination affirme librement son pouvoir créateur face aux pesanteurs du Temps et de l'Histoire. Au-delà de leur ancrage historique dans les débats idéologiques d'une époque, heureusement dépassés, les textes «conjecturaux » de Rosny proposent ainsi une méditation poétique sur l'Histoire et les pouvoirs de l'Ecriture, qui conserve tout son intérêt pour le lecteur d'aujourd'hui.

\section{NOTES}

1. «Aux carrefours de la nouvelle : Rosny aîné, poète de l'impur », in. A. Huftier (éd.), La Belgique : un jeu de cartes? De Rosny aîné à Jacques Brel, Lez Valenciennes, $n^{\circ} 33$, Valenciennes, Presses Universitaires de Valenciennes, 2003, p. 37.

2. Voir ibid., p. 54. Voir, également, E. Lysoe, "Rosny aîné ou le fantastique à pied d'œuvre ", in A. Huftier (éd.), Rosny aîné \& les autres formes, Otrante, n¹9-20, Paris, Kimé, 2006, p. 59.

3. Le terme métissage est, en toute rigueur, réservé aux croisements entre " races " ou variétés intra-spécifiques, les croisements entre espèces étant désignés par celui d'hybridation. Cette distinction a cependant peu de pertinence ici, surtout si l'on tient compte du sens très large que donne Rosny au mot « race».

4. Voir, p. ex., D. Couégnas, « Préhistoire et récit " préhistorique » chez Rosny et Wells ", Europe, n 681-682, janv.-fév.1986, p. 26, 27.

5. Voir, p. ex., Eyrimah, IIIe part, chap. V (RP, p. 195), Nymphée, ch. VIII (RSF, p. 231).

6. Nous empruntons ce terme à M. Guillaumie, Le Roman préhistorique. Essai de définition d'un genre, essai d'histoire d'un mythe, Limoges, PULIM, 2006, p. 186, n. 1, qui distingue le racialisme, défini comme une idéologie fondée sur " l'idée fausse suivant laquelle l'espèce humaine se diviserait en races, qui seraient des acteurs de l'histoire ", du racisme proprement dit, constitué, selon lui, à la fois par «un ensemble disparate de théories sur les prétendues races humaines » et par «une pratique d'exclusion qui ne s'embarrasse pas de théorie ».

7. Voir, par exemple, M. Bulliard, L'Enjeu des origines: Les romans préhistoriques de J.-H.Rosny aîné, Lausanne, Archipel, coll. «essais », 2001, p. 85-86, qui estime que, pour Rosny, le métissage constitue «l'aboutissement » du processus de moralisation de l'être humain, dans la mesure où « il suppose de la part de ceux qui s'y prêtent un renoncement définitif à l'identité propre de leur 
race, à ce pourquoi leurs pères se sont battus durant tant de siècles, pour adopter l'attitude la plus confraternelle qui soit ».

8. Op. cit, p. 135,305 . Nous voudrions marquer ici notre dette à l'égard de cet ouvrage, dont nous reprenons un certain nombre d'analyses et de suggestions dans notre travail.

9. Par récit "conjectural», nous entendons toute œuvre de fiction décrivant un monde « différent » de celui du lecteur, qu'il soit situé dans le présent, le passé ou le futur, et construit par extrapolation à partir d'un savoir scientifique, réel ou hypothétique, qui sert de caution à l'affabulation narrative. Ce qui nous a conduit à regrouper dans un même corpus récits de «merveilleux scientifique », fictions " préhistoriques » et récits de «mondes perdus ». Nous en avons exclu, par contre, des récits « d'aventure exotique », comme Le Trésor dans la brousse ou Le Lion.

10. Liste des abréviations et des références: L'Etonnant Voyage de Hareton Ironcastle (LVHI), Paris, J.-J. Pauvert, coll. « Indes noires », 1965 ; « La Fille bleue » (LFB), in Rosny aîné \& les autres formes, Paris, Kimé, 2006, p. 275-278 ; «La Résurrection de mon oncle Jérôme » (LROJ) , in La Belgique : un jeu de cartes, p. 65-70 ; La Sauvage aventure(LSA), Paris, Albin Michel, 1935 ; Les Femmes de Setné (LFS) , Paris, Ollendorf, 1903; Les Navigateurs de l'infini (LNI), Paris, Hachette, coll. «Le Rayon fantastique ", 1960 ; Récits de science-fiction (RSF), édition établie et préfacée par J-B Baronian. Introduction de J. Van Herp, Verviers, A. Gérard/Marabout, 1973; Romans préhistoriques (RP), édition préparée et présentée par J.-B. Baronian, Paris, R. Laffont, coll. « Bouquins », 1985.

11. Ce texte est, comme on le sait, une version abrégée de Vamireh.

12. Nous avons regroupé sous cette dénomination les textes relevant du genre des «mondes perdus », au sens strict, et ceux appartenant au genre des « lost race stories », cette distinction, par ailleurs utile, n'ayant que peu d'intérêt par rapport à notre propos.

13. Ce roman constitue une version plus développée et sensiblement modifiée des Hommes sangliers. Le principal changement apporté concerne le dénouement, beaucoup moins dramatique que dans la nouvelle, puisque la jeune femme enlevée et violée par les Hommes-Kérabaus survit finalement à ses épreuves et trouve le bonheur auprès du fils d'un colon hollandais.

14. Voir, p. ex., M. Bulliard, L'Enjeu des origines, p. 84-86, p. 101-104. Sur le thème du métissage dans le roman " préhistorique » en général, voir les analyses suggestives de M. Guillaumie, op. cit ., p. 146-147, 186-187.

15. IIIe part., chap. V-VI (RP, p. 306-311).

16. Ce qui reste vrai, même lorsque le récit comporte un épisode violent comme c'est le cas dans La Fille bleue, où la mort de la jeune indigène est racontée sur un ton plutôt léger, presque humoristique ( Voir, p. ex., p. 278 : «tu as commis une gaffe homicide », " Puisqu'on te donnait la petite... ce n'était pas pour des prunes », «Les Hommes bleus étaient de braves types, au fond, encore qu'ils n'eussent pas pour la vie humaine un respect exagéré »).

17. Voir, notamment, RSF, p. 106-107, 109-110.

18. Voir, p. ex., LSA, éd. A. Michel, p. 82 : «[...] je ne serais pas étonnée que sa race n'eût aucun rapport direct avec aucune race humaine» et p. 104 «Etaient-ce positivement des hommes ou appartenaient-ils à une « espèce » incapable de se reproduire avec d'autres humains [ ...]».

19. RSF, p . 209-210, 224, 231.

20. Cf. LSA, éd. A. Michel, p. 105 : "Peut-être, dans la nuit des âges, plusieurs "espèces » humaines ont-elles paru. Peut-être les Hommes-Kérabaus descendent-ils de l'une de ces espèces ». Rosny reprend ici une thèse qu'il avait déjà développée dans ses ouvrages de vulgarisation sur la préhistoire. Voir, p. ex., Les Origines (la préhistoire), rééd., Paris, Crès, 1923, p. 28: «Parmi les anthropoïdes disparus, certaines espèces furent, vraisemblablement, à peu de chose près, aussi intelligentes que l'homme, et même on est porté à imaginer qu'il se forma dans le principe des variétés d'anthropopithèques dont quelques-uns tendirent à une humanité très différente de la nôtre [...]». Comme le rappelle M. Guillaumie, op. cit., p. 161-162, cette hypothèse polygéniste était depuis longtemps abandonnée par la science. 
21. Voir, p. ex., E. Lysoe, « Rosny aîné ou le fantastique à pied d'œuvre », in A. Huftier (éd.), Rosny aîné \& les autres formes, p. 57-60.

22. Voir, p. ex., Les Navigateurs de l'infini ou La Résurrection de mon oncle Jérôme. La remarque vaut également pour une œuvre comme Le Trésor dans la neige, où nous avons deux narrateurs homodiégétiques, l'un intradiégétique, l'autre extradiégétique.

23. Nous empruntons cette expression à Ph. Clermont, "Visions d'altérité chez Rosny aîné ", in A. Huftier (éd.), Rosny aîné \& les autres formes, p. 88. Voir également, id., « Naturalisme et pseudoréalisme du merveilleux-scientifique de Rosny aîné ", in Un seul monde. Relectures de Rosny aîné. Etudes réunies par Ph. Clermont, A. Huftier \& J.-M. Pottier, Valenciennes, Presses Universitaires de Valenciennes, 2010, p. 229.

24. Ce modèle, fondé sur une généralisation du schéma narratif mis en œuvre dans les textes "fantastiques", insiste sur l'idée de conjonction entre univers étrangers. Voir notamment, E. Lysoe, « Rosny aîné ou le fantastique à pied d'œuvre ", art. cit., p. 41-47.

25. Le roman est censé se passer au XXIe siècle. Voir LNI, IIe part., chap. IV, éd. Hachette, p. 156.

26. Sur ce point, nous nous permettons de renvoyer à notre article, « Les Mondes perdus de J.H. Rosny aîné ou l'origine introuvable », in Ph. Clermont, A. Huftier \& J.M. Pottier (éds.), Un seul monde. Relectures de Rosny aîné, p. 251.

27. Voir, p. ex., LNI, IIe part., chap. XV, p. 242, où l'un des personnages suggère que les Martiens vivent « dans un milieu qui a peut-être quelque analogie avec un milieu encore à venir sur Terre ». La Terre représente inversement, pour les Martiens, une image de leur passé ancestral. Voir, p. ex., ibid., p. 239, 242.

28. Voir, p. ex., LNI, Ière part., chap. X, éd. Hachette, p. 119.

29. Bien que Glâva descende d'une ancêtre appartenant théoriquement à une autre "race " qu'Helgvor, il est précisé que les deux « races » proviennent « d'une souche commune » (Ie part., chap. V, RP, p. 484) et sont restées très proches l'une de l'autre, notamment sur le plan culturel.

30. Voir, RSF, p. 107, 111, 112.

31. A. Gourdet, « Mélange des genres », in A. Huftier (éd.), Rosny aîné \& les autres formes, p. 104.

32. L'Ennemie, in La Résurrection, Paris, Plon, 1895, p. 86. Ce récit, qui appartient à la veine réaliste de l'auteur, contient un certain nombre de réflexions intéressantes sur le métissage comme rapport du Même et de l'Autre.

33. Sur l'imaginaire de la décadence à la fin du XIXe siècle, voir notamment, P. Citti, Contre la décadence. Histoire de l'imagination française dans le roman (1890-1914), Paris, PUF, 1987.

34. Voir, p. ex., LNI, éd. Hachette, p.66, 69, 76, 86.

35. Nymphée, Ie part., chap. IV (RSF, p. 203).

36. Pour Rosny, comme pour la plupart de ses contemporains, la civilisation est porteuse par ellemême de décadence. Voir, p. ex., Les Origines, op. cit., p. 153 : « [...] le développement de l'esprit, le développement surtout des facultés de raffinement, mène à la mort des races ».

37. Sur ce motif narratif, fréquent dans les récits de "mondes perdus", voir A.-M. Boyer, "Mondes perdus, cités oubliées et retrouvées », in Mondes perdus, Textes réunis et présentés par A.-M. Boyer, Modernités, nº 3, Bordeaux, Presses Universitaires de Bordeaux, 1991, p. 12, 15-16.

38. Voir, p. ex., Vamireh, chap. IX (RP, p. 50-51), Le Trésor dans la neige, chap. IV (RSF, p. 330), LNI, Ie part., chap. VII (éd. Hachette, p. 88-89)

39. D'où une certaine forme d'anthropocentrisme, d'ailleurs dénoncée par Rosny lui-même (cf. LNI, Ie part., chap. III, éd. Hachette, p. 61), qui n'est que la traduction en termes «biologiques » d'un universalisme moral étendu aux dimensions de l'ensemble de l'univers. Cet universalisme « cosmique » apparaît comme l'un des thèmes majeurs de la pensée de Rosny.

40. Cette dimension morale est particulièrement sensible dans Les Navigateurs de l'infini, où l'on peut relever un certain nombre de passages anticolonialistes. Voir, p. ex., LNI, IIe part., chap. IV, éd. Hachette, p. 156-157. Pour une interprétation critique de ces passages, voir R. Bozzetto, 
« Rosny et ses chimères », in. A. Huftier (éd.), La Belgique: un jeu de cartes ?l, p. 34-35, qui y voit surtout l'expression d'une mauvaise conscience devant la réalité coloniale.

41. Sur cette distinction, voir, plus haut, n. 8 .

42. Un autre monde (RSF, p. 46-47), Le Trésor dans la neige (RSF, p.344, 363), La Jeune vampire (RSF, p.434-439), Les Navigateurs de l'infini, IIe part., chap. XV-XVI et Epilogue (éd. Hachette, p. 245-252), Dans le monde des variants (RSF, p.111-112).

43. Le seul autre exemple que l'on puisse citer est celui d'un personnage très secondaire de La Sauvage aventure, un serviteur d'Hendrik De Ridder, décrit comme un «bâtard de Chinois et d'Hindou » (éd. A. Michel, p. 9)

44. Sur ce passage, voir M. Guillaumie, Le Roman préhistorique, p. 142-143.

45. Sur ce point, voir, M. Bulliard, op. cit., p. 77-101; R. de Felici, Le Roman préhistorique de J.-H. Rosny aîné, Rende, Università della Calabria, Centro Editoriale e Librario, 2006, p. 82-87, 236-239.

46. Rosny reprend ici sous une forme romancée la théorie dite du « hiatus » défendue à la fin du XIXe siècle par G. de Mortillet. Rapidement contestée, elle est aujourd'hui totalement abandonnée. Voir A. Leroi-Gourhan, Dictionnaire de la préhistoire, Paris, PUF, coll. "Quadrige », 1997, s.v. « Hiatus », p. 510b et 512a.

47. IIIe part., chap. II, RP, p. 550 .

48. Helgvor du fleuve bleu, IIIe part., chap. II (RP, p. 542).

49. IIIe part., chap. III, RP, p. 553.

50. Le même phénomène apparaît dans Le Lion (1908), un roman « d'aventure exotique », où le narrateur, d'origine européenne, s'unit à une jeune femme arabe, qui lui donne deux enfants «l'un bistré et aquilin», «l'autre blond et presque rose» (in Nymphée, Société française d'imprimerie et de librairie, Paris, 1909, p. 329-330)

51. Ou, du moins, d'une « race » extrêmement proche. Voir, plus haut, n. 29.

52. Voir, Ie part., chap. I, RP, p. 456.

53. LNI, p. 171 n. 1 : «Les filles tripèdes peuvent concevoir sans le concours du mâle. Il suffit que tous deux le désirent pendant quelque temps avec intensité » et p. 242 : «Rappelez-vous que les filles martiennes peuvent devenir mères par elles-mêmes lorsqu'elles le désirent pendant longtemps et avec une grande intensité ...»

54. Cet autre mode de reproduction est évoqué dans la Ière partie du roman : « Leur étreinte, car leur acte nuptial est une étreinte, semble extraordinairement pure. C'est tout le corps qui aime, en quelque sorte immatériellement. Du moins, si la matière intervient, ce doit être sous la forme d'atomes dispersés, de fluides impondérables" (LNI, Ie part., chap. VII, éd. Hachette, p. 87). Le narrateur et Grâce connaissent une étreinte de ce genre (voir ibid., p. 120) mais elle reste sans conséquence.

55. RSF, p. 439

56. Ce que l'on pourrait symboliser par les deux formules suivantes : (MEME + AUTRE) + AUTRE ${ }^{\circledR}$ AUTRE ( $1^{\text {ère }}$ hypothèse) et (MEME + AUTRE) + AUTRE ${ }^{\circledR}$ AUTRE + AUTRE (2ème hypothèse).

57. RSF, p. 344, 347.

58. RSF, p. 363.

59. Ibid.

60. Sur ces relations triangulaires, voir A. Gourdet, art. cit., p. 104, qui propose cependant une interprétation sensiblement différente de la nôtre, dans la mesure où elle s'attache d'abord au rôle de médiateur de l'Autre par rapport aux deux autres éléments de la relation.

61. Voir, LNI, IIe part., chap. XVI (éd. Hachette, p. 244-245), Epilogue (éd. Hachette, p. 251-252).

62. RSF, p. 409.

63. A la proposition de fonder sur Terre une petite colonie martienne, «inoffensive par définition» (LNI, IIe part., Epilogue, éd. Hachette, p. 251), l'un des compagnons de Jacques Laverande fait cette réponse significative: «Ce sera peut-être inoffensif [...] mais enfin, ce pourrait être aussi un péril ! La Terre a singulièrement ranimé Grâce et le Chef Implicite » (ibid., 
p. 252). Cette idée est encore soulignée dans le paragraphe final, où, après avoir évoqué «[l']atmosphère de douceur» qui enveloppe les personnages et les "paysages de Mars se mêl [ant] aux sites de la montagne estivale » (ibid.), le narrateur décrit la lutte féroce de différentes espèces végétales, s'exterminant mutuellement.

64. Comme c'est le cas chez Gobineau, par exemple, dont la philosophie de l'Histoire s'articule autour de l'idée d'une "dégénérescence » de l'Humanité, liée au métissage. Sur la question du métissage chez Gobineau, voir P. Tort, La Pensée hiérarchique et l'évolution, Paris, AubierMontaigne, coll. « Résonnances ", 1983, p. 198-257.

65. Nous apprenons en effet que Touanhô « s'est fort bien adaptée à une vie plus confortable » ( RSF, p. 362) dans la maison d'Alglave, où le couple mène « une vie simple et belle [...] la vie des ancêtres de la Madeleine, sauf le luxe d'une demeure bâtie, de quelques meubles, de quelques aliments de choix, de café et de vin » (ibid., p. 363).

66. Rosny évoque souvent, dans ses fictions "préhistoriques» comme dans ses récits de «mondes perdus », l'idée d'une «jeunesse du monde » et de l'espèce irrémédiablement perdue. Sur ce point, voir, p. ex., R. de Felici, Le Roman préhistorique de J.-H. Rosny aîné, p. 74-77, M. Guillaumie, Le Roman préhistorique, p. 183-186, L. Guillaud, « Les Mondes perdus de Rosny aîné : la régression et le sacré », in A. Huftier (éd.), Rosny aîné \& les autres formes, p. 226-228.

67. L'exemple qui s'impose immédiatement ici est celui de L'Etonnant voyage de Hareton Ironcastle. Sur la pratique de «l'hybridation » dans ce roman, voir, p. ex., B. Lecigne, « Le Voyage littéraire de Hareton Ironcastle », Europe, n 681-682, janv.-fév. 1986, p. 89-95; H. Desmarets, « D’un horizon à l'autre : L'Etonnant Voyage de Hareton Ironcastle » in A. Huftier (éd.), La Belgique : un jeu de cartes ?, p. 155-170 ; J.P. Picot, « L'Etonnant voyage de Hareton Ironcastle : un hapax générique », ibid., p. 171-192.

INDEX

Mots-clés: J. H. Rosny, roman préhistorique, science fiction, mondes perdus, racisme, métissage

\section{AUTHOR}

\section{FRANÇOIS LAFORGE}

Ancien élève de l'Ecole Normale Supérieure de la rue d'Ulm. Agrégé des lettres. A publié différents articles sur le roman français, du XVIIIe siècle au XXe siècle (Robert Challe, Sénac de Meilhan, Flaubert, George Sand, Vallès, Queneau, Marcel Aymé). Ses dernières publications portent sur l'œuvre conjecturale de J. H. Rosny aîné et les rapports de sa pensée et de son imaginaire avec la science de son époque (« Les ‘ mondes perdus' de J. H. Rosny aîné ou l'origine introuvable ", in Un seul monde. Relectures de Rosny aîné, éd. Ph. Clermont, A. Huftier et J.-M. Pottier, Valenciennes, Presses Universitaires de Valenciennes, 2010, p. 241-263 ; « Race, histoire et décadence dans les récits ' préhistoriques’ de J. H. Rosny aîné ", Belphégor, Vol. X, n², 2011). 